\title{
How ACM Evolves in Response to Community Needs
}

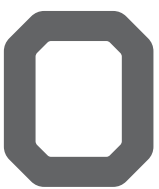

NE QUESTION PEOPLE ask me as President is how ACMas a global, volunteer-based organization-can evolve over time. We have all seen new publications, conferences, SIGs, and chapters added to the ACM family as technology grows. But what about the organization itself? Does it also evolve to keep up with changes going on in our profession?

The basic structure of ACM doesn't change frequently, but it does get adjusted from time to time in response to new priorities and needs. If you are not familiar with ACM's governance structure, there are four boards that manage our core products and activities: Publications, Education, Practitioners (Lifelong Learning), and SIG Governance. A system of regional councils was created to respond to emerging needs in different geographic areas (currently Europe, China, and India). This year, ACM has added the concept of thematic councils, which leverage products and activities from all the boards and regional councils to address important challenges for our profession.

\section{Ensuring Technology Reflects Its Full Audience}

One key to ACM's future health is to ensure its governance and activities involve people from diverse backgrounds. Diversity is defined very generally to encompass not just physical characteristics like gender, age, race, and disabilities, but also characteristics related to a person's career, such as institution type and size, disciplinary domain, workplace role, stage in career, and location where an individual resides. Ideally, ACM would be balanced in terms of the individuals, institutions, and disciplines represented.
However, our members, volunteers, and activity participants are self-selecting. We can aim to be proactive-and more effective-in recruiting volunteers from among diverse audiences, but most of our efforts need to focus on inclusion. That is, we must try to become more diverse by creating environments that are welcoming of new and perspectives, and where hostility and other antisocial behaviors are not tolerated.

If you have registered for an ACM conference in the last couple of years, you have seen that we have an organizationwide Policy Against Harassment at ACM Activities (http://bit.ly/2LcV88z), which lays out the expectations for professional behavior at all ACM activities, including committee meetings. In addition, many ACM's SIGs have programs that specifically address diversity. These include awards, travel grants, accessibility reviews, targeted mentoring, fellowships, and competitions to promote broader participation in computing. In July, a new Diversity and Inclusion Council was established to identify "best practices," document them, and encourage their broader adoption across the organization. Led by co-chairs Natalie Enright Jerger and John West, the Council will also reach outside ACM to find new strategies and partnerships that can help improve diversity in the broader community.

\section{Taking Responsibility for Technology We Create}

You already know about the ACM Code of Ethics and Professional Conduct (www.acm.org/code-of-ethics), which has received a lot of attention since the updated version was released last year. The changes reflect the fact that for some time now, ACM has been discussing what kinds of ethical and so- cial responsibilities we should take for the technology we create and deploy. You have only to look at past issues of $\mathrm{Com}^{-}$ munications to find that the risks and challenges have been discussed in virtually every issue in recent years.

Technology, developed and deployed around the globe, is at the heart of some of the most pressing issues we face as a society. Concerns about data breaches, election security, digital privacy, surveillance, and the future of the Internet stretch beyond national borders and go to the heart of how we live, work, and interact with one another. As a professional society whose members are at the forefront of developing these technologies, ACM has had active policy forums in the U.S. and Europe for many years. These activities have been instrumental in providing technical expertise and advice to policy-makers at a variety of levels. In July, we established a global Technology Policy Council to strengthen and extend ACM's policy efforts even further. Led by Lorraine Kisselburgh, it brings together leading experts to lend a global perspective to the social and ethical challenges posed by technology. One of their first initiatives is to develop a new series of bulletins presenting a balanced perspective on the impact of specific developments or applications of technology, targeted not just at decision-makers but also our general membership and the press.

I hope you are as excited as I am about these two new initiatives. To keep up on what they are doing or to send your own ideas, visit the ACM website.c

Cherri M. Pancake is President of ACM, professor emeritus of electrical engineering and computer science, and director of a research center at Oregon State University, Corvallis, OR, USA.

Copyright held by author/owner. 